\title{
ESTUDIO COMPARATIVO DE LA ÉTICA ANTE LO NUEVO EN LA INVESTIGACIÓN ACCIÓN Y EL MAOÍSMO
}

\author{
Miguel Angel Olivo Pérez* \\ https://orcid.org/0000-0002-4571-6972 \\ Juan José Abud Jaso** \\ https://orcid.org/0000-0002-5207-4082
}

RECIBIDO: Octubre 2019 / ACEPTADO: Abril 2020 / PUBLICADO: Mayo 2020

\begin{abstract}
Como citar: Olivo Pérez, Miguel; Abud Jaso, Juan. (2020). Estudio comparativo de la ética ante lo nuevo en la investigación acción y el maoísmo. Telos: revista de Estudios Interdisciplinarios en Ciencias Sociales, 22 (2), Venezuela. (Pp.410-428).

DOI: www.doi.org/10.36390/telos222.12
\end{abstract}

\section{RESUMEN}

En el presente artículo, se expone un estudio comparativo de corte cualitativo, de dos formas de indagación grupal: a) La investigación acción, cuyo pionero fundador fue Kurt Lewin (1973) en Estados Unidos, misma que posteriormente sería desarrollada en el campo de la educación (Stenhouse, 2007, Elliot, 2000, 2018, Shön, 1992, Brockbank y McGill, 2002, Wenger, 2001, Wittrock, 1989), b) El maoísmo, que tuvo su origen en la doctrina de Mao Zendog promovida durante la Revolución China (Paternostro, 1972, Mehnert, 1973, Nee y Peck, 1975), así como durante la Revolución Cultural China (Piao, 1975; MacFarquhar y Schoenhals, 2009). El propósito de la investigación fue estudiar y evaluar el componente ético de ambas, entendido como la capacidad de hacer frente a lo nuevo (Badiou, 2010a), donde de manera potencial surgen o se reformulan determinados compromisos y figuras de autoridad. Para ello, se siguió la metodología de comparación de tendencias espontáneamente desarrolladas, lo cual se dio desde el diseño organizativo de un mismo punto de partida en el tiempo. Es decir, como primer paso se organizó un solo curso taller con alumnas educadoras que trabajan con niños de nivel preescolar, donde se promovió la indagación acerca de las intervenciones a niños con alteraciones en su desarrollo. $Y$ como segundo paso, se fueron realizando observaciones etnográficas a lo largo del proceso destinadas a obtener datos sobre los compromisos y figuras de autoridad. Del proceso surgieron dos subgrupos: uno más próximo al maoísmo y el otro a la investigación acción. Las aportaciones destacan la importancia de la ética frente a lo nuevo, desde un fuerte compromiso en la resolución de los problemas, lo cual exige trascender la

\footnotetext{
* Profesor en la Universidad Pedagógica Nacional, Doctor en Ciencia Social con Especialidad en Sociología, Miembro del Sistema Nacional de Investigadores nivel II. Autor del libro El impasse del sujeto. Badiou, la sociología y la modernidad. México. Correo electrónico: miguelangelolivo@hotmail.com

** Doctor en Filosofía y psicoanalista. Especialista en teoría crítica y hermenéutica. Profesor en la Facultad de Filosofía y letras de la Universidad Nacional Autónoma de México (UNAM) y en la Universidad Católica Lumen Gentium. Autor del libro Las rebeliones en el pensamiento. México. Correo electrónico: kabirabud@hotmail.com
} 
obediencia mecánica a la norma, para en cambio aceptar plenamente la autoridad emergida del exceso del encuentro amoroso.

Palabras clave: educación, investigación acción, maoísmo, ética, autoridad.

\section{Testing Ethics in Action Research and Maoism: A Comparative Study}

\section{ABSTRACT}

Following a qualitative method, this paper compares two forms of group investigation: a) action research, whose founding pioneer was Kurt Lewin (1973) in the United States, and later developed in the field of education (Stenhouse, 2007, Elliot, 2000, 2018, Shön, 1992, Brockbank y McGill, 2002, Wenger, 2001, Wittrock, 1989), b) Maoism, which had its origin in the Mao Zendog doctrine promoted during the China's Revolution (Paternostro, 1972, Mehnert, 1973, Nee y Peck, 1975), as well as during the Chinese Cultural Revolution (Piao, 1975; MacFarquhar y Schoenhals, 2009). The purpose was to study and evaluate its ethical component, understood as the ability to deal with the new (Badiou, 2010a), where potential commitment and authority figures arise or are reformulated. To achieve this, the methodology of comparison of trends is used to study different courses of events spontaneously developed, starting from the same starting point situation. In other words, as a first step, a single workshop was organized with female educators working with pre-school children, where research on interventions for children with developmental disorders was promoted. And as a second step, ethnographic observations were made throughout the process to obtain data on commitments and authority figures. Two subgroups emerged from the process: one closer to Maoism and the other to action research. The contributions highlight the importance of the ethics of dealing with the new, based on a strong commitment to solving problems in the care of children, which requires transcending the mechanical obedience to the norm, to instead fully accept the authority emerging from the excess of loving encounter.

Key words: education, action research, maoism, ethics, authority.

\section{Introducción}

Habiendo surgido durante los años 1930's - 1940's la investigación acción y el maoísmo comparten una historia en común escasamente conocida, en tanto que ambas se proponen como formas, alternativas a la ciencia, de poner en interacción las ideas y la práctica. La investigación acción se plantea como un modelo de intervención social basado en la indagación colectiva de las situaciones que subyacen a determinados problemas sociales, con el fin de solucionarlos recuperando las mejores ideas disponibles (Lewin, 1973; Suárez, 2002; Goyette y Lessard-Hebert, 1988). Ello, en el contexto de la psicología social estadounidense en ese entonces en boga.

Posteriormente, hacia los años 1940 la propuesta de Lewin se extendió como forma de atender diferentes problemáticas sociales en los Estados Unidos (p.e. el desempleo, el 
alcoholismo, el embarazo adolescente, la violencia, etc.). Fue sólo hasta los años 1990, con los planteamientos de diversas perspectivas emergentes de la investigación acción (Stenhouse, 2007, Elliot, 2000, 2018, Shön, 1992, Brockbank y McGill, 2002, Wenger, 2001, Wittrock, 1989), que se constituyó como una definida corriente de estudios en el campo de la educación y la pedagogía en Estados Unidos y posteriormente en varios otros países de occidente.

Por su parte, el maoísmo nace durante la revolución China de los años 1930's, como propuesta de pensamiento y práctica sobre la contradicción del líder Mao Zendog (1968a, p. 333). Posteriormente, con el advenimiento de la revolución cultural en dicho país hacia 1966 y 1967, el maoísmo se difundiría de manera extensa como forma de educación a través de la organización de decenas de miles de grupos de indagación que operaban en diferentes lugares y maneras, ya sea como comités revolucionarios en las escuelas e instituciones, o bien en los distritos urbanos, como grupos obreros de propaganda, comités de partido, la educación de campesinos, o comités de producción en las fábricas (Paternostro, 1972, Mehnert, 1973, Nee y Peck, 1975). Tales prácticas inspiraron a movimientos de izquierda en varios países, principalmente de Europa (Gerd-Rainer, 2007; Bourseiller, 1996; Cardina, 2013; Dressen, 1999).

Ya sea en sectores o instituciones específicas, o como parte de las luchas políticas más amplias, el caso es que durante los años 1930's-1940's se recurrió a la discusión colectiva en pequeños grupos, como forma de retroalimentar la teoría y la práctica, con miras a enfrentar las tensiones de la época. La investigación acción y el maoísmo tienen en común lo siguiente:

1) Son modelos de intervención vigentes, en el sentido de que en la actualidad llegan a ser empleados en diferentes contextos.

2) Tienen en común el objetivo de educar a través de la investigación tomando en cuenta las situaciones que se enfrentan.

3) Persiguen la retroalimentación entre la teoría y la práctica. No se limitan a la mera aplicación de la teoría, sino que persiguen el conocimiento concreto del objeto enriquecido de la manera más plena. La objetividad de lo investigado se logra a través de la realización: "emplear la investigación significa realizarla" (Stenhouse, 2007, p. 38). Por su parte en el maoísmo el objetivo de la investigación se alcanza mediante "el análisis concreto de situaciones concretas" (Bosteels, 2005, p. 579).

4) Están diseñados para ser aplicados en la vida cotidiana. Para el maoísmo: "La revolución es en la vida y transforma la vida" (Bosteels, 2005, p. 590). Por su parte realizar investigación acción exige la indagación rigurosa en estrecho contacto con los problemas que surgen en lo cotidiano.

5) Se caracterizan por ser formas de organización abiertas al cambio, en el sentido de pretender modificar según las circunstancias, tanto las formas de coordinación de las acciones como los contenidos y objetivos que le dan sentido a los esfuerzos grupales. Como se verá más adelante en la parte metodológica, dicha característica de apertura al cambio, fundamenta la pertinencia de estudiar comparativamente el maoísmo y la investigación acción, a partir no tanto de un diseño experimental, como de uno centrado en dinámicas de tendencias y posibilidades.

En las actuales concepciones de la ética, las actitudes y acciones ante lo nuevo, han sido objeto de mayor reflexión (Badiou, 2010a, Groys, 2016), fenómeno que puede ser analizado en concreto a través de los grupos de indagación. En un nivel y en un lenguaje abstractos, Badiou (2002, p. 58) refiere del siguiente modo lo real del momento ético decisivo: "toda idea es 
el ser actual de una propiedad, y el Bien no indica ninguna, puesto que es aquello a partir de lo cual toda propiedad, toda idea, llega a la potencia de la partición que ella misma instituye".

En el corazón de las propuestas de la investigación acción y el maoísmo, se encuentra presente la idea hegeliana de Aufhebung: la superación de tensiones llevadas hacia un nuevo nivel. En Lewin la superación es vista como un logro grupal, obtenido gracias a que fuerzas opuestas pueden ser estabilizadas a través de un determinado arreglo entre los participantes (Billig, 2015). En cambio, en Zendog la superación es concebida como el modo en que las contradicciones en el seno del pueblo son resueltas a través del empleo de la dialéctica en la política (Zendog 1975, p. 143). A nivel de los pequeños grupos maoístas, se procuran superar no sólo las contradicciones internas, sino también las externas. Dicha necesidad provenía de la exigencia maoísta de considerar simultáneamente la universalidad y la particularidad de las contradicciones (Zendog cit. en Fan, 1970, p. 18).

Ya sea que se busque un nuevo equilibrio, o se pretenda llevar las contradicciones a un nuevo nivel, de cualquier manera, en ambos casos la presentación de lo nuevo conlleva una frontera móvil, donde los dilemas de la libertad y la igualdad impactan "sin cesar en la repartición de lo político y lo social" (Bensaïd, 2010, p. 51). En otras palabras, ya sea a nivel de un Estado o de un pequeño grupo de personas, la libertad se encuentra en relaciones de tensión específicas con la igualdad, donde la política, entendida como "producción de un determinado efecto" (Bensaïd, 2010, p. 35), se entrelaza con formas concretas de relación social, de manera que es posible valorar la ética por la forma en que las personas enfrentan sus problemas. Es en este sentido, y a partir de las premisas teóricas que se presentan a continuación, que es posible comparar la forma en que la ética es puesta a prueba

\section{Marco teórico. La ética en la investigación acción y el maoísmo}

Retomar la línea de reflexión que sigue Bensaïd acerca de la repartición de lo político y lo social, exigiría extender la reflexión sobre lo grupal, hacia la problemática del Estado como instancia ética, en tanto se recurre al derecho del discernimiento 0 a la deliberación, como medios de actualización de los ideales políticos. Sin embargo, en vez de desarrollar la reflexión teórica a través de las aporías de la democracia y sus contrarios, implicadas en las cuestiones de lo político y lo estatal, y con el fin de centrar la atención en la dimensión ética de la indagación grupal, aquí interesa enfatizar el potencial de transgresión ínsito en el acto grupal de proponer problemas e intentar solucionarlos en grupo.

En consecuencia, desde el momento en que la identificación y la superación (Aufhebung) de los problemas, se plantea como la tarea central de la indagación grupal, la idea de transgresión puede potencialmente entrar en escena. Cuando se habla de transgresión también se habla de autoridad. Es decir, en los grupos de indagación, con la necesidad de enfrentar lo nuevo con los propios recursos grupales, junto con la de valorar el grado en que se han de expandir la libertad y la igualdad, se hace inevitable la pregunta "¿Hasta dónde debo obedecer a la figura de autoridad?" Ello, ya sea de manera explícita en los discursos hablados, o de manera implícita a través de la puesta en juego del significante emanado de la figura de autoridad en cuestión.

No se puede descartar tampoco la posibilidad de que la presentación del Aufhebung se de cómo necesidad de ganancia absoluta (Žižek, 2015), en la que la valoración de la expansión de la libertad y la igualdad no se limita a simples contrastes de formas de ejercer la 
política ya sea desde diferentes ideologías, naciones o formas de organización grupal, sino que dicha ganancia de infinito actual realizada a partir de acontecimientos singulares, puede manifestarse en modalidades muy heterogéneas. Esto significa que la investigación acción y el maoísmo, lejos de ser meros procedimientos formales de grupo, en sus aplicaciones concretas para la resolución de problemas, pueden volverse indistintos en tanto ambas formas de indagación grupal pueden potencialmente servir como matemas de pase (Miller, 1987); es decir, como exigencia radical de cumplimiento de un principio incondicional, mismo que puede derivarse de la presentación de una verdad sustractiva (Badiou, 2003).

Con la presentación de la verdad sustractiva y la exigencia de un cambio de coordenadas de la situación en forma de necesidad de una superación (Aufhebung) de los problemas, lo otro aparece como indeterminado. En tales situaciones, los imaginarios y símbolos en torno a una determinada figura de autoridad son puestos en juego, por lo que determinados límites marcados por dicha figura devienen en susceptibles de ser transgredidos por parte de los participantes.

Sin embargo, aunque por un lado la aplicación de los grupos de indagación en las escuelas se plantea como una formidable oportunidad para impulsar el cambio social en algún nivel de la realidad social, por el otro lado esta óptica contrasta radicalmente con respecto a la que suele predominar en el magisterio de educación básica en México acerca del comportamiento ético, comúnmente entendido en el medio como el seguimiento de acciones de acuerdo con normas.

Otro aspecto que es necesario considerar cuando se da la posibilidad de transgredir fronteras impulsando determinados cambios, es la tendencia a la relajación que durante las últimas décadas ha experimentado la figura de la autoridad en las escuelas y en las familias: ésta ya no se presenta en figuras definidas ni en encarnaciones específicas, sino que se han vuelto más sutiles, de manera que en la transición desde la escuela tradicional hacia la escuela moderna, los ejes de las figuras de autoridad tradicionales se diversificaron y desplazaron hacia otras figuras menos autoritarias (Zamora y Zerón, 2009). Ello, además de adquirir un carácter mucho más sutil, en donde las circunstancias, tanto como las figuras internalizadas de autoridad, son mucho más móviles, y por lo tanto la ética ya no funciona bajo el canon de una norma, sino como una disposición que sólo sale a la luz en momentos cruciales en que determinados dilemas morales son puestos en juego.

Así, desde el momento en que las indagaciones y acciones grupales son lanzadas en forma de apuestas que se ambicionan congruentes, tales apuestas pueden tener diferentes alcances en sus resoluciones logradas, así como en la eficacia de los medios y la aproximación a los fines perseguidos. Estas tres dimensiones; a saber, los problemas, los medios y los fines, pueden ser evaluadas desde el punto de vista moral.

Cabe destacar que no se trata de una simple evaluación de la desviación con respecto a normas. Más fundamentalmente, la evaluación incluye la consideración de los engaños, manipulaciones y otras perversiones de diferentes tipos, a que los problemas, los medios y los fines son sometidos, donde con gran frecuencia los individuos son utilizados de manera particularista en desmedro de valores universales que desde Aristóteles en occidente se conocen como valores propios de universales in re (Casin, 2018). A partir de las consideraciones anteriores, el problema es el del rescate de la investigación acción y el maoísmo, como dos formas de indagación grupal potencialmente actualizables en su ética. 
Ahora bien, el proceso de intentar superar problemas al momento de enfrentar a lo nuevo, y con ello también la posibilidad de transgredir determinados límites y figuras de autoridad, nunca se dan en un vacío abstracto, es decir, en ausencia un contexto histórico de contenidos específicos. Por lo que es necesario especificar los respectivos contextos relativamente generalizados en los países antes mencionados, en que las indagaciones grupales propios del maoísmo y la investigación acción, ponen en relieve sus compromisos en resolver los problemas que se proponen, en donde eventualmente reformulan los límites entre lo permitido y lo prohibido, así como reconfiguran sus imaginarios y relaciones con la autoridad.

\section{El maoísmo}

Desde veinte años antes de que el Partido Comunista ascendiera al poder en China en 1949, la propuesta de Mao Zendog para organizar grupos de reflexión y praxis, se fue aplicando como una manera de hacer la revolución a través de la aplicación de los principios marxistas leninistas. Así por ejemplo en 1941, Mao enfatizaba la necesidad de una mayor sistematización de la investigación y el estudio de la realidad objetiva que enfrentaban (Zendog, 1968b, p. 14). Ello, en crítica a la negligencia que advertía en sus camaradas, en el estudio de la historia y la situación concreta que en ese entonces vivían tanto dentro como fuera del país. Exhortaba por lo tanto, a evitar caer en los dogmas, los sectarismos, el teoricismo, el subjetivismo, el individualismo y el particularismo, identificando y corrigiendo los errores al tiempo de permanecer en estrecho contacto con las masas, así como confrontando constantemente los conocimientos con la experiencia. En especial para el objetivo que interesa al presente trabajo, Mao destacaba la cualidad de los nuevos cuadros en tanto poseen un agudo sentido de lo nuevo (Zendog, 1968b).

Años después, durante la revolución cultural China en los años 1966 y 1967, Mao exhortó a sus millones de seguidores a desburocratizar el Partido Comunista (Piao, 1975; MacFarquhar y Schoenhals, 2009). Lejos del supuesto de que se trataba de un dictador dando consignas a millones de peleles obedeciendo ciegamente, lo que tuvo lugar allí fue un amplio movimiento de masas en torno a Mao y su doctrina. Las universidades devinieron en un medio propicio para una movilización sin precedentes, donde millones de jóvenes estudiantes, y poco después de obreros y campesinos, se dedicaron efervescentemente a estar atentos, reflexionar y discutir confrontando el libro rojo de Mao con lo que sucedía a su alrededor, y más en general, con su país:

"los grupos, comités y congresos de la revolución cultural son las mejores formas de organización mediante las cuales las masas se educan a sí mismas [...] no deben ser organizaciones provisionales, sino organizaciones de masas permanentes y verdaderas. Son adecuadas no sólo para las escuelas y las instituciones, sino en lo fundamental también para las fábricas, minas y otras empresas, para los barrios y aldeas" (Partido Comunista Chino,1978, p. 27).

La revolución cultural ofreció la oportunidad a millones de personas tanto en la propia China como en varios otros países del mundo, para incorporarse a la original secuencia política que unificaba a las masas a manera de relativizar el poder del Estado. En palabras de Badiou (2010b, p. 3), maoísta francés y uno de los filósofos más prestigiados en nuestros días: 
"Toda clase de trayectorias subjetivas y prácticas encontraron, en la incansable actividad de los revolucionarios chinos, su nominación. Cambiar de subjetividad, vivir de otro modo, pensar de otro modo [...] al final de los años sesenta estábamos por todas partes, en las fábricas, en las ciudades, en el campo. Decenas de millares de estudiantes se convertían en proletarios, o vivían en las residencias obreras. También existían, para esto, palabras de la Revolución cultural: los "grandes intercambios de experiencias", "servir al pueblo", y, siempre esencial: la "ligazón de masas" [...] El pequeño libro rojo de Mao era nuestra guía, no para todo, como dicen los necios, con fines de catequización dogmática, sino muy al contrario, para aclararnos e inventar vías nuevas en toda clase de situaciones dispares anteriormente desconocidas para nosotros".

Reconociendo el profundo impacto existencial que la revolución cultural China tuvo en cientos de miles de jóvenes estudiantes, obreros y campesinos, Bosteels señala: "Pocos comentaristas fallan en reconocer la sorprendente expansión con la cual el campo de juego político fue tema hacia fines de los sesentas y principios de los setenta" (Bosteels, 2005, p. 589). En específico, lo que cabe rescatar de dicha experiencia, es que la transgresión se dio a través de la forma de un desplazamiento de la figura de autoridad del Partido Comunista hacia el propio líder Mao.

Los acontecimientos posteriores, de regresión a la vía capitalista, demostrarían que en el fondo se trataba de una división del Partido-Estado (Badiou, 2010b, p. 10), una división que convoca a la reflexión. Como lo demuestran otras experiencias, a nivel de los grupos la figura de la autoridad no sólo puede estar afectada por una división fundamental, sino también por su ausencia: por ejemplo, la del pueblo nómada caminando en círculos en torno a determinada región, cuyo rey recién había muerto, pero era más poderosa la necesidad de continuar la vida como si nada hubiese pasado y todo siguiera igual; es decir, en la lógica de defender su monarquía religiosa dando vueltas en círculos con el cadáver del rey, y sin que el pueblo lo supiera (Geertz, 1994). Así, es en torno a la pregunta de cómo la ética se pone a prueba ante la posibilidad de modificar el arreglo de la relación con la autoridad, que cabe estudiar y comparar cómo se da este fenómeno al interior de los grupos de investigación acción y el maoísmo.

\section{La investigación acción}

Desde su nacimiento en el contexto liberal estadounidense durante la década de los 1940 's, la investigación acción se presentó como un medio a través del cual es posible "lograr en forma simultánea avances teóricos y cambios sociales" (Salazar, 1992, p. 15). Sin embargo, con el paso de los años Lewin le fue dando mayor importancia a la elaboración teórica que al trabajo con grupos sociales concretos. Así, no es gratuito que él haya sido también autor de la teoría de campo en psicología social (Lewin, 1939). A diferencia del maoísmo, la investigación acción se propone como una herramienta o un modelo que supuestamente puede ser empleado cualesquiera que sean los fines que sus usuarios se propongan e independientemente del contexto en que se encuentren inmersos. Es decir, se concibe como neutralmente valorativa y por ende como si estuviera más allá de cualquier ideología. Sin embargo, en sus aplicaciones por lo general se limita al contexto de las aulas escolares (p.e. ver Schein, 1996) las escuelas y 
las empresas (p.e. ver Cummings, et. al. 2015), excluyéndose el contexto político, social y económico más amplios del país o el entorno internacional en que se proponen.

Desde el momento en que la investigación acción es considerada como una herramienta o un modelo, en su concepción y puesta en práctica se puede advertir la tendencia a enfatizar las formas en detrimento de los contenidos. Lo cual sucede al punto de quedar subsumida por una pedagogía de los procedimientos como contenidos (Pozo y Postigo, 2000). Aún más, en el ambiente actual de la ideología tecnocrática imperante en los sistemas educativos latinoamericanos, donde la moda de concepciones como las competencias promueven la formación de cuerpos dóciles (Zabala y Sánchez, 2019), las propuestas de investigación acción tienden a ser vaciadas de significado, a manera de no pocas veces verse reducidas a ser empleadas bajo una lógica instrumental, al considerárseles como simples medios estratégicos eficaces para obtener determinados logros cuantitativos, desvirtuándose y eliminándose así por completo, los tintes ligeramente radicales que tuvieron en sus primeras aplicaciones como forma de atender a grupos vulnerables de la sociedad estadounidense.

Durante las décadas de los 1970's y 1980's en América Latina, surgieron propuestas un tanto menos conocidas, pero sin embargo vigorosas, de recuperación de la investigación acción para fines de cambio social radical en la región, denominadas con el término Investigación Acción Participativa (Stavenhagen, 1992; Fals, 1980; Zamosc, 1987), las cuales se asemejan en varios aspectos a los grupos maoístas al reivindicar el empoderamiento de los grupos vulnerables del pueblo. Tales planteamientos recuerdan también a la propuesta de actor colectivo de Touraine (1987), la de grupos de discusión de (Ibañez, 1992) y las comunidades ideales de habla de Habermas (1987).

Se trata de propuestas que asumen explícitamente el objetivo de la emancipación, y por tanto, se encuentran abiertas a nuevas nominaciones, mismas que al igual que el maoísmo, se ocupan más por abordar el análisis del contexto inmediato y amplio, característica que los distancia de la investigación acción, más inclinada a incurrir en el riesgo de un teoricismo que discurre de manera distanciada del contexto en que sus reproductores viven.

En efecto, así como en los 1930's los psicólogos de la New School defendían la psicología de la Gestalt al tiempo que mostraban su desdén por el psicoanálisis (Jay, 1991, p. 224), y en los 1970's parte de la izquierda francesa asimilaba el maoísmo con entusiasmo (Bosteels, 2005), de manera similar en cada latitud y época, tanto en la academia como entre los legos, se van tomando decisiones acerca de los enriquecimientos semánticos que en su turno se consideren más viables para cada opción política, en la esperanza de que, como señala Marramao (1989, p. 17), los conceptos se encuentren en condiciones de "producir relaciones, de modelar y orientar sus propios referentes socio-políticos".

Una de las recreaciones más significativas que se han dado en América Latina de los grupos de indagación orientados a la emancipación, fue la de la Investigación Acción Participativa, de quien interesa aquí recuperar, de uno de sus máximos exponentes, Fals Borda (1980), tres dimensiones de su propuesta, con el fin de enriquecer las expresiones empíricas del trabajo de campo que atañe a la presente investigación. Cabe señalar que, si bien tales recuperaciones no hacen justicia a la riqueza y enormes aportaciones de este autor y luchador social, vale la pena recalcar la importancia de profundizar en esta valiosa corriente de pensamiento en trabajos posteriores más amplios. Las tres dimensiones que interesa recuperar de la misma son: 
a) El despliegue de las reflexiones colectivas en la lógica de la emancipación, se dan a partir de las peculiaridades en que prevalecen determinadas estructuras de desigualdad socioeconómica y política en nuestra región latinoamericana.

La consideración de tales peculiaridades reviste una importancia de primer orden, considerando que ellas condicionan las maneras de pensar y las oportunidades para el cambio, no sólo en una lógica de sobrevivencia, sino también bajo una lógica de emancipación.

b) El rescate de la memoria histórica acumulada de las luchas contra la desigualdad en el pasado.

Si bien por un lado la memoria histórica permite darnos cuenta de que existen determinadas persistencias en las luchas contra la desigualdad que pueden ser valiosas considerar, por el otro lado, es menester tomar en cuenta también la permanente posibilidad del gesto divisor en momentos cruciales de la historia; decir, la identificación del problema ético implicado en las nuevas situaciones (Badiou, 2002), mismas que plantean la elección irreductible entre renunciar 0 comprometerse en tal lucha.

c) El impulso a la participación más amplia e igualitaria posible.

Tanto en el maoísmo como en la Investigación Acción Participativa y demás propuestas de emancipación, esta premisa reviste una importancia central no sólo debido al imperativo ético del empoderamiento de los grupos de indagación, sino también porque la consideración de la igualdad no puede ir desligada de la libertad: las situaciones abiertas al cambio ponen en juego determinadas figuras de autoridad que es menester analizar en sus dinámicas concretas.

Como puede advertirse, estas tres características centrales de la Investigación Acción Participativa, al igual que el maoísmo y demás propuestas de emancipación desde los grupos de indagación, a partir del momento en que privilegian la praxis transformadora, se encuentran abiertas a su manifestación concreta in situ. Para el caso que aquí atañe, el de la investigación acción y el maoísmo, importa profundizar en particular, en el análisis empírico de los compromisos y figuras de autoridad puestos en juego en situaciones donde se da la posibilidad de lo nuevo, sin que ello signifique desconocer la memoria histórica, ni la importancia de la participación igualitaria.

\section{Metodología}

A nivel empírico, el estudio comparativo que a continuación se presenta entre la investigación acción y el maoísmo, parte de la premisa de que los grupos de indagación en general, al hacer frente a nuevas situaciones y problemas, se ven necesariamente expuestos a ir sobre la marcha cambiando tanto sus formas de organización, como la manera en que conciben los contenidos de la realidad. Es decir, al ser ejercidos en la práctica, adquieren dinámicas concretas que rebasan cualquier modelo formal, y por lo tanto, enfocarse en el estudio de las posibilidades y tendencias de su desenvolvimiento, se vuelve más importante que atender a modelos formalmente concebidos, basados en unos pocos indicadores fijos, incapaces de captar el carácter inmanente de las realidades grupales.

Para fines de la comparación empírica, de índole cualitativa, de la ética en la investigación acción y el maoísmo, interesa destacar aquí los contenidos y posibilidades de desenvolvimiento que en concreto adquieren dos variables estrechamente relacionados con la ética de hacer frente a lo nuevo: el compromiso de la atención a los niños y las figuras de 
autoridad. Los datos fueron recopilados a través de la observación participante en un curso taller práctico reflexivo de intervenciones de alumnas educadoras, a niños de preescolar con problemas en su desarrollo.

Como es de esperarse comúnmente en los grupos escolares, conforme transcurrieron las sesiones se fueron distinguiendo tres subgrupos de acuerdo a los grados de compromiso de atención a los niños (dos de los cuales sirvieron para la comparación empírica de la investigación acción y el maoísmo que más adelante se detalla). El grupo global estuvo constituido en total por veintidós alumnas de unos de los grupos de la Licenciatura en Educación Preescolar con apoyo de las Nuevas Tecnologías de la Información y la Comunicación (LEPTIC) de la Unidad 096 de la Universidad Pedagógica Nacional (UPN), de México, a quienes se les extendió la invitación a formar parte de la indagación grupal, cada una seleccionando un niño con alguna alteración en su desarrollo para intervenirlo en sus propios lugares de trabajo.

Acorde con los principios de la Investigación Acción Participativa, de propiciar la mayor participación y discusión posibles, con dicha invitación, se procuró informarlas y garantizarles el máximo de libertad para decidir por sí mismas sus propias acciones de intervención, con base en las discusiones que ellas mismas iban proponiendo y el facilitador organizando a la vez que registrando, con el fin de que tuvieran claras sus opciones.

Es decir, a diferencia de los grupos de Lewin y Mao, no se dio por supuesto el predominio de la figura de un líder (que en este caso supuestamente sería el docente del grupo), equívoco en el que ciertamente incurrió Kurt Lewin (Billig, 2015), sino que con el llamado a exponer sus puntos de vista libremente, la cuestión de las figuras de autoridad subyacentes en sus concepciones y prácticas se mantuvo abierta a su libre manifestación durante el curso y posterior estudio en gabinete.

El grupo de indagación se reunió una vez a la semana; a saber, cada sábado, en doce sesiones de una hora y media cada una. Sus intervenciones a los niños se realizaron en las mismas escuelas de preescolar donde ellas laboran, de lunes a viernes de 9 AM a 2 PM, mismas que fueron reportadas en reflexiones escritas que ellas realizaron tanto en sus hogares, como en las sesiones sabatinas de aula en la Universidad Pedagógica Nacional. En éstas últimas, las alumnas educadoras también participaron a través de reflexiones y discusiones que externaron de manera oral en cada una de las sesiones de aula.

Gráfico 1. Organización del trabajo de campo de la investigación

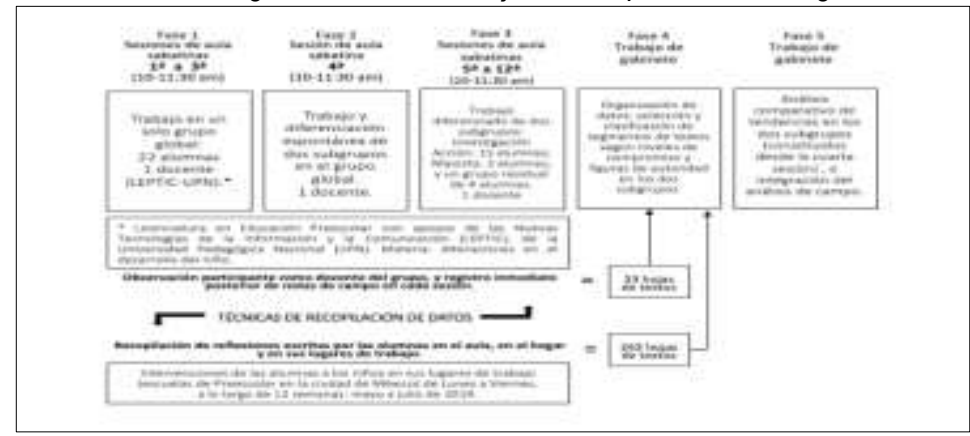

Fuente: Elaboración propia. 
Cabe señalar asimismo, que la igualdad en las condiciones de participación, sólo estuvo restringida o condicionada por la historia grupal previa, en la cual no se observaron restricciones grupales que causaran sesgos significativos.

En cada sesión, durante la primera media hora se discutía y valoraba hasta qué punto era posible detectar e intervenir exitosamente a los niños. Posterior a ello, cada una escribía sus experiencias de la semana en una hoja amarilla. Asimismo, durante su tiempo en casa tuvieron como cometido, escribir en hojas azules sus experiencias, mismas que entregarían al final del curso.

A la cuarta sesión sabatina del taller, como ya se esperaba, pudo distinguirse a las alumnas más comprometidas, mismas que en lo posterior irían a conformar un primer subgrupo, a partir del cual sería comparado el segundo subgrupo. Dicha comparación reviste una importancia crucial, dado que constituye el objeto principal de la investigación empírica que aquí se desarrolla. La diferencia entre este subgrupo que para los propósitos de la presente investigación fue denominado maoísta y los demás, se manifestaba en: a) el tiempo que dedicaban a la intervención a los niños, y de suyo, en el compromiso con ellos, b) el mayor interés que prestaban al contexto más amplio que rodeaban a sus intervenciones.

Así, es a partir de haberse evidenciado tales diferencias, que pudieron distinguirse al interior del grupo global de las veintidós alumnas tres subgrupos, los cuales para fines de la presente investigación se denominaron: subgrupo maoísta, subgrupo de las formales de investigación acción y subgrupo residual. Tales subgrupos se fueron distinguiendo poco a poco durante las tres primeras sesiones, para surgir de manera más definida y espontánea a la cuarta sesión, a manera de facilitar en lo posterior las comparaciones pertinentes. Cabe señalar por su parte, que el subgrupo residual integrado por cuatro alumnas, no fue incluido como objeto de estudio, debido a que su nulo interés por intervenir a los niños, imposibilitó contar con los datos necesarios que pudieran servir de material para el respectivo análisis de campo.

\section{Fundamentos y desarrollo de la comparación}

El subgrupo maoísta, integrado por tres alumnas que comenzaron a sobresalir en el grupo global, participaba con mayor intensidad y calidad tanto en las discusiones grupales, como en sus trabajos escritos. Se planteaban preguntas certeras y densas; su espíritu de disquisición tendía a elevarse notablemente con respecto al de sus demás compañeras. Hacia la mitad del curso habían trazado ya un pequeño historial de interacciones entre ellas, a partir de las cuales retroalimentaban sus experiencias y aprendizajes, las cuales no se limitaban al aula, sino que iban más allá de ella.

Este fenómeno ocurrió de tal modo, que en lo sucesivo se irían consolidando como un subgrupo emergente distintivo. Para los integrantes de este subgrupo lo que estaba en juego era mucho más que un esfuerzo plasmado en un simple trabajo para entregar al maestro o para participar en clase: se trataba del amor a la vocación, nacido de una concientización de la situación ética ante los niños.

Así, por ejemplo, una de ellas refirió la experiencia de haberse atrevido en una ocasión a jugar con los niños gateando por entre las bancas y debajo de las mesas a pesar de sus más de cien kilogramos de peso, lo cual arroja como un significativo dato, la gran energía que puede desarrollarse y canalizarse como vocación de educadora a partir de la relación emocional que se establece con los niños. Otra de ellas aludió a la circunstancia al inicio del curso, de haberse 
arriesgado valientemente a aceptar dentro de uno de sus cursos regulares, a un niño con un defecto de nacimiento en su rodilla, cuyos ligamentos tenían alta probabilidad de romperse en movimientos bruscos. Afortunadamente dicho niño pudo terminar el preescolar exitosamente sin que hubiera ocurrido nada grave con su delicada condición física.

A grosso modo puede considerarse que la suma de los diferentes momentos a partir de los cuales las alumnas educadoras decidieron participar cada vez más intensivamente a través de las discusiones y los escritos, es lo que centralmente constituyó a este subgrupo de tres integrantes, cuyos rasgos característicos se aproximan a lo que aquí se ha denominado maoísmo, razón por la cual le llamaremos subgrupo maoísta.

Muy diferentes fueron las características de las demás alumnas del grupo, de entre quienes pudieron distinguirse a su vez dos subgrupos: uno no integrado y más bien disperso, formado por las alumnas que menos interés mostraron en las actividades a lo largo del curso; a saber, cuatro. Puede decirse que este último subgrupo se distinguió más bien por su mera agregación sumatoria, es decir, disperso, sin lazos sociales significativos entre sí, y por lo tanto sin significantes claros con las cuales poder adjetivarlas fuera de su bajo compromiso en sus intervenciones, razón por la cual se le denominó subgrupo residual.

El otro subgrupo formado por quince integrantes se distinguió a lo largo del curso por apegarse a las formalidades de lo que desde un principio interpretaron como una vida escolar normal: asistir a las sesiones, participar con algunos comentarios o reflexiones, así como elaborar y entregar los trabajos a tiempo. Dada esta característica central, y las siguientes que veremos enseguida, a este subgrupo se le denominó subgrupo de las formales de investigaciónacción.

El sentido del "nosotros" del subgrupo de las formales, si bien había surgido ya desde dos años atrás durante las interacciones en sus cursos previos en la Universidad Pedagógica Nacional, también se fue formando de manera peculiar y espontánea a través del curso taller de intervención a los niños con alteraciones en su desarrollo. Específicamente, el sentimiento del "nosotros" en este grupo, se erigió casi exclusivamente para cumplir con la tarea concebida como "asignada" por el docente facilitador, lo cual se dio más por costumbre que por una auténtica iniciativa de apropiación participativa relativamente profunda. Sus integrantes tendieron a cumplir sólo de manera formal con las discusiones e intervenciones: sus alusiones a los procedimientos institucionales y normativas escolares eran frecuentes.

De manera semejante, acudiendo a estereotipos en lugar de a singularidades, calificaban generalizadoramente a sus niños de "inquietos", con "problemas de comportamiento", "sin límites", etc. A este subgrupo se le puede considerar como de investigación acción no sólo porque su visión se limitaba al aula y al niño considerado individualmente, sino además porque sus intervenciones se reducían a retroalimentar la práctica y la reflexión a una acción vista y emprendida predominantemente como rutina en el marco de las actividades escolares convencionales.

\section{Resultados}

Dado que las probabilidades de intervenir a los niños a partir de un auténtico y profundo compromiso, reviste una importancia crucial para la averiguación de la ética en los grupos de indagación, fue menester centrarse como uno de los principales resultados de esta investigación comparativa, en la distancia que separa al subgrupo de las formales de investigación acción del 
subgrupo maoísta en dicho compromiso, así como de las figuras de autoridad que subyacen a cada subgrupo.

Las alumnas integrantes del subgrupo de las formales de investigación acción, sólo eventualmente mostraron autenticidad tanto en las intervenciones como en las discusiones grupales. Un ejemplo entre algunos otros de esto último, puede advertirse en la experiencia referida por una de sus integrantes acerca de un niño que, acostumbrado a la indisciplina, una día se le detectaron muchos piojos en su cabeza, por lo que discretamente se le regresó a su casa para después, al día siguiente llegar feliz a la escuela con el cabello rapado abrazando a la educadora, actitud que sorprendió gratamente a esta y comenzó a ver con nuevos ojos al niño.

Días después le llego a ver, de manera inesperada, muy atento, equilibrado y servicial, para con un niño compañero suyo con síndrome de Down. A partir de entonces, la educadora pudo acompañarle en un proceso de crecimiento mucho más satisfactorio que en días anteriores. A través de las anteriores experiencias, pudo advertirse la capacidad de dicha educadora para la intervención ante situaciones nuevas. Sin embargo, en términos de dedicación y capacidad para identificar las situaciones problemáticas y aproximarse en su resolución, se distanciaba considerablemente de cualquiera de las tres integrantes del grupo maoísta.

De manera semejante, la mayoría de las educadoras del grupo de investigación acción (de doce a catorce) en diferentes momentos aislados llegaron a realizar abundantes y detalladas descripciones, mismas que aunque por su amplitud no pueden ser presentadas en este espacio, sin embargo sirvieron como referentes suficientes para afirmar que dicho subgrupo no pudo arribar a una resolución en sus intervenciones; es decir, no fueron capaces de detectar la pauta de intervención apropiada para el niño en cuestión, a manera de alcanzar la superación (Aufhebung) cualitativa de su situación de intervención, en el sentido en que la entiende Žižek (2015, p. 523), como la contingencia elevada a la forma de la necesidad, que hace de la excepción una situación singular a partir de la cual se da la adhesión a un compromiso universalista.

Sin embargo, mayormente las actitudes de compromiso manifestadas por este subgrupo, fueron en buena medida causadas por el subgrupo maoísta. Es decir, fueron infundidas desde una instancia externa que, de manera semejante a la respuesta ante la exhortación a intervenir a los niños, tuvo un carácter superficial y por ende, la opción a comprometerse a profundidad en la atención a los niños, fue experimentada por ellas más bien como coercitiva.

A diferencia de las integrantes del subgrupo maoísta, las de este grupo por varias razones renunciaron a dar el paso decisivo hacia un mayor compromiso. Algunas veces era miedo a confrontar recuerdos amargos; otras, más frecuentes, simplemente no entendían una exigencia escolar nueva a la que no estaban acostumbradas; otro motivo, de índole estructural, es que solían estar demasiado ocupadas en su triple jornada, es decir: labores de amas de casa, trabajadoras y estudiantes, de manera que, o bien renunciaron a desplegar mayores esfuerzos a ésta última, o sintieron que la exigencia como estudiantes afectaría sus compromisos con las otras dos.

Tanto o más significativos para la presente investigación, fueron los motivos de renuncia a un mayor compromiso, por la situación de confusión a la que al menos dos terceras 
partes de estas alumnas, es decir, al menos diez, ingresaron durante las reflexiones grupales, fenómeno ante el cual, lejos de apresurarse a ser considerado como una consecuencia de falta de organización del aprendizaje, debe de ser visto como una parte necesaria de la naturaleza de los grupos de indagación que se pretenden realizar con una alta participación de sus integrantes. De acuerdo con el teorema del punto de exceso (Carvalho y França, 2019, p. 4), siempre hay sub-múltiples, mismos que aun cuando se encuentran incluidos en una situación, no son ni pueden ser numerables en dicha situación. Se presenta un irremediable exceso de sub-múltiples.

En consecuencia, en tales situaciones, las incertidumbres ante la proliferación de temas de diversa índole y la densidad de las reflexiones, asi como numerosas contradicciones o tensiones, propiciaron en las alumnas educadoras la sensación de estar desubicadas, toda vez que no se encontraban en un curso escolar convencional, y por lo tanto tenían ante sí varias cosas nuevas a las que se les hizo difícil hacer frente, sobre todo en el marco de la dinámica y exigencia predominante generada por el subgrupo maoísta, que planteaba la necesidad de reconvertir el excedente, según entiende este término Dólar (2017, p. 22), de los sentimientos hacia los niños, en un encuentro amoroso con ellos.

Tal desorientación experimentada por la mayoría del subgrupo de las formales, resulta comprensible considerando que, en los grupos de indagación signados por una alta participación, las reflexiones dialécticas desplegadas con el objetivo de distinguir entre la esencia y apariencia, así como entre lo que pertenece a una situación y lo que no pertenece a la misma, requieren de habilidades arduamente ejercitadas para estar lo suficientemente formadas. Bosteels (2005), refiere del siguiente modo la experiencia de ruptura con la realidad inherente al maoísmo: "no es menos cierto que cada estructura de los lugares asignados está siendo constantemente transformada como resultado de las divisiones interiores, rupturas y cambios". (p.600)

La complejidad derivada de la variedad de puntos de vista vertidas en las discusiones en el grupo global, demuestra que la consolidación de la indagación grupal requiere mucho más de doce sesiones. La dificultad para afrontar tal situación crece con mayor razón, cuando lo que se encuentra en juego, es el dilema ético entre escoger un mayor o un menor compromiso en la atención a los niños. Sin duda el problema planteado a las alumnas es profundo, pues atañe directamente a la revalorización de su trabajo propio, algo a lo que no se encuentran acostumbradas a ejercer, dadas las dificultades implicadas en el desarrollo de una profesión que cotidianamente se realiza en la precariedad y con un bajo reconocimiento y estatus (Olivo, 2014), pues con frecuencia es considerada una actividad reducida a, como se dice coloquialmente en México "cuidar niños y cambiar pañales".

A partir de lo anteriormente observado en el subgrupo de investigación acción, se puede afirmar que la fuerte adhesión a los convencionalismos en las formas acostumbradas de participar en el aula, donde cotidianamente el ejercicio de revalorización inmanente de la profesión de educadora es ausente, tienden a inhibir e impedir más que a facilitar, la asunción del riesgo de modificar varios aspectos de la situación.

A su vez, no es gratuito que tales rasgos de comportamiento se hayan reproducido en la mayor parte de las integrantes del grupo global: al comportarse de acuerdo con las reglas ya aprendidas e instaladas como reglas del juego en el aula, los automatismos se encuentran a la orden del día. En comparación con el subgrupo maoísta, en el subgrupo de las formales de la 
investigación acción, la disposición a romper con varias formas acostumbradas de organizar la vida diaria en la familia y la profesión, y por lo tanto la capacidad de mostrar una ética ante lo nuevo, fue considerablemente menor (ni se diga del grupo residual).

En los casos de los compromisos medios y bajos del subgrupo de las formales de la investigación acción y del subgrupo residual, las resistencias a realizar una descripción densa (Geertz, 1992) de sus intervenciones era formidable. Sólo lo hacían de manera escueta y sin comunicar demasiado sus afecciones relacionadas con el proceso de intervención. Tal fenómeno sin duda se encuentra estrechamente relacionado con los imaginarios y significantes de autoridad que operan en sus vidas en general y no sólo en la escuela.

Como se señaló anteriormente en el marco teórico, en las últimas décadas se ha verificado un relajamiento, así como un desplazamiento y diversificación de las figuras de autoridad (Zamora y Zeron, 2009), de manera que a nivel empírico se hace necesario indagar las pulsiones y los significantes subyacentes a los semblantes 0 apariencias primeras en la decisión de optar por una vía de acción y no por otra. En este caso, ya sea por la investigación acción o el maoísmo.

En las alumnas educadoras de las formales de investigación acción, pudieron identificarse dos figuras de autoridad: a) la figura de la madre protectora y b) el automatismo reflejado en la pulsión de insertarse en un orden determinado, en el cual las cosas que transcurren todos los días son altamente predecibles, o en otras palabras, en ésta última la inclinación a tomar riesgos ante lo nuevo es considerablemente inhibida.

Si bien la primera encarna en un rol social más definido que en la segunda —la cual se encuentra más diluida en la conciencia y opera más a nivel inconsciente-, ambas nociones de autoridad se insertan en sus propias peculiares correspondientes maneras en la investigación acción y en el maoísmo. En este último subgrupo, la autoridad que se manifiesta como una pulsión, lo hace a través de la adhesión a principios que pueden llegar a rozar el fanatismo y con ellos, sesgos en la forma de concebir la realidad objetiva, como por ejemplo: "los niños están muy mal en nuestra sociedad y hay que salvarlos", "casi ningún adulto de hoy está capacitado para cuidar a un niño o ser buen padre o madre", "me siento muy sola en el mundo enfrentando estos problemas", etc. Por su parte la autoridad que se manifiesta en la figura de la madre protectora, aunque puede llegar a ser más poderosa que la anterior, no está exenta del peligro de mezclarse con fanatismos como el anteriormente señalado.

En el subgrupo de la investigación acción, la autoridad como pulsión incurre en el peligro de seguir procedimientos sin cuestionarlos ni razonarlos, incurriendo con ello en una intervención instrumental, gestionaría y falta de amor (Cueva, 2007, p. 259). Aquí conviene poner la mirada más allá del aula, pues el problema no reside en una defensa del compromiso con la escuela o las demandas de los docentes, sino con la sociedad y en este caso, con los mismos niños con alteraciones en su desarrollo.

Así, seguir procedimientos por pura convención, aunque el contexto señale vías más eficaces para la intervención a los niños, puede llegar a ser una vía muy riesgosa. Por ejemplo, se llegaron a dar casos de educadoras que, al enterarse de casos de abuso sexual a un menor, eluden el sentido común al atenerse a rígidos protocolos que seguidos sin sensibilidad resultan, en el menos peor de los casos, absurdos, y en los peores, extremadamente dañinos. Vale la pena señalar que tal sesgo en la intervención puede resultar incluso más perjudicial que la misma alteración que se atiende en el niño (Olivo, 2013, p. 153). 
En cuanto a la autoridad encarnada en la figura de la madre protectora y amorosa, puede afirmarse que las integrantes del grupo de investigación acción, aunque privilegiaron el aspecto formal y convencional de "cumplir con las tareas", sin embargo detectaron de inmediato el carácter sui generis del curso, lo cual significa que hacia la cuarta sesión, tomaron la decisión consciente de distanciarse de las prácticas del grupo maoísta; es decir, no es tanto que hayan sido incapaces de comportarse amorosamente con los niños, sino que en ellas la fuerza de las convenciones y las formalidades pesaron más que el compromiso incondicional en la atención a los niños, lo cual a su vez implicaba convertirse en sujeto de enunciación (Sañudo y Susinos, 2018), a través tanto de los trabajos escritos como de las participaciones orales. Esto último exige una conexión existencial, misma que si bien comprendieron como presente en las integrantes del grupo maoísta y estuvo a su disposición efectuarla, tomaron la "libre" decisión de seguir adheridas a las convenciones de aula.

Tal decisión la tomaron aún a sabiendas de que pudieron optar por aprender de las intervenciones cobijadas bajo la figura de madre amorosa para su profesión, pues prefirieron limitar a dicha figura al ámbito del hogar, lo cual no forzosamente equivale a que en otros determinados momentos futuros más favorables, puedan rescatarla del valioso reservorio cultural familiar mexicano prevaleciente en los sectores populares de la población (Caballero, et. al. 2014), como sí se atrevieron a hacerlo las del grupo maoísta.

\section{Discusión y conclusiones}

Dado que el objetivo principal de las comparaciones fue evaluar la ética ante lo nuevo, en tanto se ponen en juego determinados compromisos y figuras de autoridad, resulta pertinente valorar la distancia entre los dos subgrupos emergidos en el proceso. Esto significa en otras palabras, que el grupo de la investigación acción fue leído y estudiado con relación a los parámetros del grupo maoísta, en especial, en lo referente a la gran riqueza de disposiciones en este último, emanadas de su compromiso en la intervención a los niños.

Quizás una de las más refinadas y profundas maneras de expresarse acerca de la ética ante lo nuevo, sea concebirla como un exceso del encuentro. Aunque en el presente trabajo las descripciones empíricas acerca de dicho exceso se hayan expuesto de manera breve, sin embargo, fue posible y suficiente presentar unos pequeños atisbos acerca del mismo, manifestados en el subgrupo maoísta. Aunque este subgrupo también puede denominarse como "Investigación Acción Participativa", es muy importante concebir a tales variaciones de indagación grupal como abiertas a nuevas nominaciones, pues lo que importa es la sustancia enfocada a una visión emancipadora.

Si bien en la actualidad los grupos de indagación en general, más allá de que sean denominados de una u otra manera, no figuran entre los mejores candidatos para albergar en su seno la gracia de la ética del encuentro, es decir, de la ética ante la cosa insondable del acontecimiento, siempre es posible reconocer que, en buena medida, ello ha sido porque nuestra mirada no está entrenada para detectarla cuando llega a darse. Sin embargo, el solo hecho de enterarnos de la posibilidad de dicho entrenamiento, y mejor aún, de su operación efectiva en miles de aulas anónimas a pesar de todo lo que juega en contra de dicha ética, motiva el optimismo suficiente para inaugurar nuevas y enriquecedoras rutas para la investigación de los grupos en donde surgen los excesos del encuentro ético. 
Aunque no se haya observado directamente, es viable suponer que no es sólo el docente, sino también diferentes posibles figuras de autoridad (p.e. el maestro, la jefa directora de sus escuelas de preescolar, la madre o el padre, etc.), quienes en peculiares mezclas ínsitas en las disposiciones subjetivas, motivan las acciones de las educadoras, ejercen sobre las mismas una influencia que va desde un compromiso superficial hasta un compromiso profundo por la obtención de logros o la solución de problemas.

De acuerdo con lo que se pudo advertir a través de la detección de las intervenciones sensibles sobre los niños sufrientes, no es el maestro ni ningún otra la figura de autoridad la que puede aportar el mayor compromiso posible de la educadora en la intervención de los niños, sino la figura madre protectora y amorosa que han internalizado, la cual se expresa en el amor al socorrer y acudir en alivio al sufrimiento de los niños. La primera consecuencia de haber detectado esta figura de autoridad en los dos subgrupos, fue que la figura del docente se relativiza, y por lo tanto, la visión exigida a los integrantes de los grupos de indagación, va más allá del aula a manera de extenderse también a los problemas surgidos en las familias propia y también en las ajenas, los cuales por supuesto, también se relacionan de complejas maneras con las figuras de autoridad en el lugar de trabajo.

En tales momentos de relativa incertidumbre ante la relativización de la figura del docente en el aula, el sentimiento de la condición de igualdad resulta crucial no sólo para la disposición a la libre expresión, sino tanto o más importante aún, para la creación de una solidaridad grupal que sirva de fuente a un creciente compromiso. A su vez, dicha solidaridad, posee como valioso potencial, servir como aliciente para la acción cotidiana militante en favor de los destinatarios finales de la educación, que en este caso fueron los niños con alteraciones en su desarrollo.

\section{Referencias bibliográficas}

Badiou, Alain (2002). Tratado de ontología transitoria. Gedisa. España.

Badiou, Alain (2003). Conferencia acerca de la sustracción. Condiciones, Siglo XXI. México.

Badiou, Alain (2010a). La filosofía, otra vez. Errata naturae. España.

Badiou, Alain (2010b). La revolución cultural ¿La última revolución? Omegalfa. España.

Bensaïd, Daniel (2010). El escándalo permanente. Agamben, G., Badiou, A., Bensaïd, D. Democracia ¿en qué estado? Prometeo. España.

Billig, Michael (2015). Kurt Lewin's Leadership Studies and His Legacy to Social Psychology: Is There nothing as Practical as a Good Theory? Journal for the theory of Social Behaviour. Vol. 5, No 4. Estados Unidos (Pp. 440-460). Extraído de https://onlinelibrary.wiley.com/doi/10.1111/itsb.12074 consulta: 19/06/19.

Bosteels, Bruno (2005). Post-Maoism: Badiou and politics. Positions: east asia cultures critique. Volume 13, Number 3. Estados Unidos (Pp. 575-634).

Bourseiller, Christophe (1996). The Maoists: The Mad History of the French Red Guards. Plon. Francia.

Brockbank, Anne y McGill, lan (2002). Aprendizaje reflexivo en la educación superior. Morata. España.

Caballero, Marta, Varela Rosa y Flores, Janette (2014). Educación en salud y desarrollo social en comunidades marginadas seleccionadas del Estado de Morelos. Universidad Autónoma del Estado de Morelos. México. Extraído de: http://riaa.uaem.mx/handle/20.500.12055/489 consulta: 09/04/2020. 
Cardina, Miguel (2013). Génesis, estructuración e identidad del fenómeno maoísta en Portugal (19641974). Revista Ayer. Vol. 4, № 92. España (Pp. 123-146).

Carvalho, Wanderley, y França, Oswaldo (2019). Singular and universal in Alain Badiou and the psichoanalysis scientificity hypothesis. Vol. 30. e180072. Brasil. DOl: https://doi.org/10.1590/0103-6564e180072

Casin, Barbara (2018). Vocabulario de las filosofías occidentales. Diccionario de los intraducibles. Siglo XXI. México.

Cueva, Marcos (2007). De la gran liberación al fin de la autoridad. Revista Mexicana de Sociología. Vol. 69, No 2. México (Pp. 243-255).

Cummings, Stephen; Bridgman, Todd y Brown, Kenneth (2015). Unfreezing change as three steps: Rethinking Kurt Lewin's legacy for change management. Human Relations. Vol. 69, No. 1. Reino Unido (Pp. 33-60).

Dólar, Mladen (2017). Uno se divide en dos. Más allá de la interpelación. Paradiso. México.

Dressen, Marnix (1999). De l'amphi à l'établi: Les Étudiants maoïstes à l'usine (1967-1989). Belin. Francia.

Elliot, John (2000). El cambio educativo desde la investigación acción. Morata. España.

Elliot, John (2018). La investigación acción en educación. Morata. España.

Fals, Orlando (1980). La ciencia y el pueblo. Asociación colombiana de sociología. La sociología en

Colombia: balance y perspectivas. Editora Guadalupe. Colombia.

Fan, Kuang-Huan (1970). La revolución cultural China. Era. México.

Geertz, Clifford (1992). La interpretación de las culturas. Gedisa. España.

Geertz, Clifford (1994). Centros, reyes y carisma: una reflexión sobre el simbolismo del poder. Conocimiento local. Paidós. España.

Gerd-Rainer, Horn (2007). The Spirit of '68: Rebellion in Western Europe and North America 1956-1976. Oxford Press. Estados Unidos.

Goyette, Gabriel y Lessard-Hebert, Michelle (1988). La investigación-acción. Sus funciones, su fundamento y su instrumentalización. Laertes. España.

Groys, Boris (2016). Introducción a la antifilosofía. Eterna Cadencia. España. Habermas, Jürgen (1987). Teoría de la acción comunicativa. Taurus, España.

Ibañez, Jesús (1992). Más allá de la sociología. El grupo de discusión: técnica y crítica. Siglo XXI. España.

Jay, Martin (1991). La imaginación dialéctica. Historia de la Escuela de Frankfurt y el Instituto de Investigación Social (1923-1950). Taurus. España.

Lewin, Kurt (1939). Field Theory and Experiment in Social Psichology: Concepts and Methods. American Journal of Sociology. Vol. 44, No 6. Estados Unidos (Pp. 868-896).

Lewin, Kurt (1973). Resolving social conflicts. Selected papers on group dynamics. Souvenir Press. Estados Unidos.

MacFarquhar, Roderick y Schoenhals, Michael (2009). La revolución cultural china. Grijalbo. España.

Marramao, Giacomo (1989). Poder y secularización. Península. España.

Mehnert, Klaus (1973). China después de la tormenta. Grijalbo. México.

Miller, Jacques-Alain (1987). Matemas I. Manantial. Argentina.

Nee, Víctor y Peck, James (1975). China's ininterrupted Revolution. From 1840 to the present.

Pantheon Books. Estados Unidos.

Olivo, Miguel (2013). Los niños vulnerables. Una perspectiva crítica. Gedisa. España. 
Olivo, Miguel (2014). La educación especial más allá del aula. Revista Internacional de Educación para la Justicia Social. Vol. 3, No. 1. España (Pp. 249-265).

Partido Comunista Chino (1978). Decisión del Comité Central del Partido Comunista de China sobre la "Revolución Cultural Proletaria. Aprobada el 8 de agosto de 1966". China. La lucha por el poder. Ediciones Ricou hacer. España.

Paternostro, Sandro (1972). La China de Mao. Mensajero. España.

Piao, Lin (1975). La revolución cultural china. Grijalbo. México.

Pozo, Juan y Postigo, Yolanda (2000). Los procedimientos como contenidos escolares. Edebe. España.

Salazar, María Cristina (1992). La investigación-acción participativa. Inicios y desarrollos. Editorial popular. España.

Sañudo, Margarita y Susinos, Teresa (2018). ¿Quién toma la palabra en la escuela?, ¿Quién escucha? Preguntas pertinentes desde la práctica reflexiva. Revista Latinoamericana de Educación Inclusiva. Vol. 12, No. 1. España (Pp. 74-94). DOI: https://doi.org/10.4067/S0718$\underline{73782018000100006}$

Schein, Edgar (1996). Kurt Lewin's Change Theory in the Field and in the Classroom. Notes Toward a Model of Managed Learning. Systems Practice and Action Research. Volumen 9, No 1. Noruega (Pp. 27-24).

Shön, Donald (1992). La formación de profesionales reflexivos. Paidós. España.

Stavenhagen, Rodolfo (1992). Cómo descolonizar las ciencias sociales. Salazar, María Cristina (1992). La investigación-acción participativa. Inicios y desarrollos. Editorial popular. España.

Stenhouse, Lawrence (2007). La investigación acción como base de la enseñanza. Morata. España.

Suárez, Mercedes (2002). Algunas reflexiones sobre la Investigación-acción colaboradora en la educación. Revista Electrónica de la enseñanza de las Ciencias. Vol. 1, No 1. España. Extraído de http://reec.uvigo.es/volumenes/volumen1/REEC 1 1 3 3.pdf consulta: 23/05/19.

Touraine, Alain (1987). El regreso del actor. Eudeba. Argentina.

Wenger, Etienne (2001). Comunidades de práctica. Aprendizaje, significado e identidad. Paidós. España.

Wittrock, Merlin (1989). La investigación de la enseñanza I, Il y III. Paidós. España.

Zabala, Josefa y Sánchez, José (2019). Evaluar por competencias o como potencia. Una mirada reflexiva y crítica. Revista Telos. Vol. 21. No 3. Venezuela (Pp. 544-563). DOI: https://doi.org/10.36390/telos213.04

Zamora, Guillermo y Zerón, Ana (2009). Sentido de la autoridad pedagógica actual: una mirada desde las experiencias docentes. Estudios Pedagógicos. Vol. 35, No. 1. Chile (Pp. 171180).DOI: http://dx.doi.org/10.4067/S0718-07052009000100010

Zamosc, León (1987). Campesinos y sociólogos: reflexiones sobre dos experiencias de investigación activa en Colombia. La investigación acción en Colombia. Foro y Punta de lanza. Colombia.

Zendog, Mao (1968a). Sobre la contradicción. Obras escogidas Tomo I. Editorial del pueblo. China. Zendog, Mao (1968b). Obras escogidas Tomo III. Editorial del pueblo. China.

Zendog, Mao (1975). Sobre el tratamiento correcto de las contradicciones en el seno del pueblo.

Cinco tesis filosóficas de Mao Tsetung. Ediciones de lenguas extranjeras. China.

Žižek, Slavoj (2015). Menos que nada. Hegel y la sombra del materialismo dialéctico. Akal. España. 